Literatur.

Das Ergebnis der Untersuchung über den Ursprung der Ministerialen geht dahin, daß sie früher Laten waren, „daB wenigstens der Hauptgrĭndungsstamm aus abhängigen, unfreien Leuten bestanden hat" (S. 52), womit also im ganzen die herrschende Theorie bestätigt wird.

Weniger glïcklich sind die Versuche Fressels, die vielbesprochene Frage zu beantworten, wie die Tatsache, daß die Ministerialen am Gericht unter Konigsbann teilnahmen, mit den Angaben des Sachsenspiegels zu vereinbaren sei. Die Seite 67 gegebene Erklärung paßt nicht; denn einmal spricht Eike in III 19 nur von des rikes dienstman, welche zur Urteilsfindung beschränkt legitimiert seien und ferner gab es im Gericht unter Königsbann überhaupt keine Vollbart des Gerichtsumstandes, an welcher die Ministerialen hätten teilnehmen können.

Den SchluB der dankenswerten Arbeit bildet der Abschnitt: die Übertragung der Gesamtrechte auf die ubrigen Lehnsleute, worin wir erfahren, daß schon zu Beginn des 13. Jahrhunderts das Lehns- und Dienstverhältnis größtenteils ineinander übergegangen waren.

Jena.

Hans Fehr.

Dr. Karl Lohmeyer: Das Hofrecht und Hofgericht des Hofes zu Loen. Ein Beitrag zur Geschichte der Münsterschen Amtsverfassung. Münstersche Beiträge zur Geschichtsforschung. Neue Folge XI. Universitätsbuchhandlung Franz Coppenrath, Münster 1906, 80 S. gr. $8^{\circ}$.

Das Buch Seeligers über die Grundherrschaft hat viel Bewegung in die Forschung über das Hofrecht gebracht, und auch der Verfasser der vorliegenden Arbeit scheint von diesem.Buche angeregt worden zu sein. Er meint, das Hofrecht von Loen erhärte den Satz Seeligers, daß das Hofrecht nur dingliche Verhältnisse regle, und betont (S.49f.), daß die Kompetenz des Hofgerichts sich nur erstreckt habe auf: Erwerb des Hofrechts, Aufnahme neuer Genossen, Auflassung von Hofgütern, Vereidigung der Besitzer und Weisung von Urteilen in Erbund Grundsachen. Es sei an der Zeit, daß mit der Ansicht "von der alles in ibre Abhängigkeit ziehenden Macht der Grundherrschaft gebrochen werde ${ }^{u}$. Das Loener Hofrecht ${ }_{n}$ ordne nur interne Hofsachen, das Gericht sei ein reines Grundgericht ohne jeden öffentlichen Charakter und die hofrechtlichen Verhältnisse bätten nicht nur einen Stand

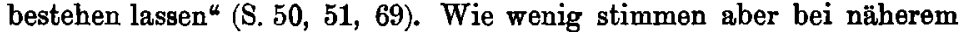
Zusehen diese formulierten Rechtssätze mit dem wirklichen Leben überein! Der Verfasser bemerkt nämlich (S. 68), daß der Grundherr die Praxis verfolgte, „die Freien bei der Übernahme eines Gutes zu zwingen, ihre Freiheit aufzugeben und als Hörige in den Verband der Hofgenossenschaft sich aufnehmen zu lassen; da $B$ man stets darauf 
bedacht war, die Hofgüter nur an solche Leute zu vergeben, die in jeder Beziohung, d. h. in allen Sachen, in denen das Hofgericht Gewalt beanspruchte, sich unterordneten (S. 69), daB in älterer Zeit alle Hofleute die Pflichten hatten, die sich auf die Hörigkeit gründeten, und daß auch noch später die Ubertragung von Gütern an Freie eine Ausnahme geblieben sei" (67). Fs scheint also hier geradezu ein typisches Beispiel für die ${ }_{n}$ alles in ihre Abhängigkeit ziehende Macht der Grundherrschaft" vorzuliegen. Die Erwerber von Hofgutern, welche ihre Freiheit aufgeben mußten, schieden eben aus dem Landrecht und dem Landgericht aus und unterwarfen sich dem Rechte des Grundherrn. Sie traten mit ihrer ganzen Persönlichkeit in den Hofverband ein und das Hofgericht des Grundherrn mußte sich auch mit ihren persönlichen Angelegenheiten befassen, kann also unmöglich auf dingliche Verhältnisse beschränkt gewesen sein. Ja, die Aufnahme von Freien in den Hofverband unter Belassung der Freiheit dürfte, nach den Ausführungen des Verfassers ${ }^{1}$ ) so sehr als Ausnahme gegolten haben, daB ich für den Hof zu Loen den prinzipiellen Satz aufstellen möchte: die Ubernahme eines Gutes hat die Unfreiheit des Beliehenen zur Folge. Das Hofrecht von Loen kennt im Prinzip nur einen Stand von Hintersassen: die Unfreien. Das wertvolle bei Ontersuchungen, wie sie Lohmeyer vorgenommen hat, liegt nicht darin, eine juristigche Konstruktion zu geben, welche den tatsächlichen historischen Vorgängen nicht gerecht wird, sondern das Rechtsprinzip zu erkennen und in den Vordergrund zu rücken, welches im wirklichen Leben zur Anwendung gelangte.

Die Arbeit Lohmeyers ist klar und prägnant geschrieben und gibt uns wichtige Aufschlüsse u. a. über folgende Punkte:

1. Über die Drucke und Handschriften des Hofrechtes, die Hofbücher, deren zwei vorhanden sind und welche die bedeutsamsten Materialien enthalten (Gerichtsentscheidungen und Weistümer) und über die Entstehung des Hofrechts, wobei zwei Hauptteile (Abschnitt 1-31 und Abschnitt 31-105) zu unterscheiden sind.

2. Untersuchungen über das Hofgericht. Dabei wird u. a. gezeigt, daß allmählich ein festes Urteilerkollegium entstand, die Tegeder oder Curgenoten oder Assessores, deren Amt mit dem Besitz eines der vier Tegederhöfe fest verknüpft war, so daß der Erbe des Hofes auch Erbe des Amtes wurde. Auch die Vollstreckung des Urteils lag in der Hand der Tegeder. Daneben waren sie Urkunds- und Aufsichtspersonen (S. 30). Für die Behauptung, daß sich neben diesem Urteilerkollegium die Gerichtsgemeinde als Urteilsfinderin aufrecht erhielt, fehlen die Belege. Auch ruht die Vermutung Lohmeyers auf

1) Die Regel, daß nur Unfreie in Hofverbande geduldet wurden, scheint so straff durchgeführt worden zu sein, daß das Hofrecht mit keinem Worte hervorhebt, in welchen Rechtsbeziehungen der Freie persönlich besser gestellt war als der Unfreie. Vgl. S. 69 A. 3. 
schwachen Füßen, daß die Institution der Tegeder in historischem Zusammenhang mit der fränkischen Dekanie stehe.

3. Mitteilungen über die Hofhörigen und die hofhörigen Güter. Hier wird u. a. der schwer zu erllärende Name Hyen erläutert. Die Hyen sind Geschworene, welche zur Besetzung des Hofgerichts hinzugezogen werden. -

Durchaus einverstanden bin ich mit dem Verfasser, daß er, entgegen der früher von Niesert ausgesprochenen Ansicht, das Eigentum an den Höfen dem Grundherrn und nicht den Hintersassen zuspricht. Die S. 76 beigebrachten Belege sind zwingend.

Jena.

Hans Fehr.

\section{Prof. Dr. Joseph Becker, Geschichte der Reichslandvogtei im Elsaß von ihrer Einrichtung bis zu ihrem Übergang an Frankreich. 1273-1648. (Straßburg 1905.)}

Die Arbeit von Joseph Becker iat die ausführlichste Monographie, die wir bis jetzt über den gesamten Verlauf der Geschichte einer einzelnen Landvogtei besitzen. Sie vereinigt im wesentlichen frühere, zerstreut gedruckte Einzelforschungen des Verfassers zu einem bequemen Ganzen, das angemessen in einen historischen und in einen systematischen Teil zerfällt. (Erster Teil. Die äußeren Schicksale der Landvogtei; Nachweis der Landvögte und Landvogteiinhaber von 12731634. - Zweiter Teil. Das Wesen und die Bedeutung des Instituts der Landrogtei.) Seiner bereits M. I. Ö. G. XXVI, 336 ff. gegen die Aufstellungen meines Buches über die Verwaltung des Reichsgrates im 13. Jahrhundert begründeten Anschauung gemäß läßt B. die Landvogtei im Elsaß durch König Rudolf I. neugeschaffen sein. So ergibt sich das Jahr 1273 als vordere Zeitgrenze, und die Zeit vor Rudolf wird nur in der Einleitung kurz behandelt. Es ist hier nicht der Ort, auf die von B. in dem genannten Aufsatz gegen mich eröffnete Polemik einzugehen, da die strittige Frage, ob es eine Landrogtei (Prokuration) Elsaß vor 1273 gegeben hat und ob sie ständig gewesen ist, mit den Mitteln eines territorial begrenzten Materiales nicht zu lösen ist, sondern erst durch die Betrachtung derselben Erscheinung in andern Landschaften Licht erbält. Ich begnüge mich deshalb, meinen abweichenden Standpunkt hervorzuheben: Ich sehe in der Prokuration wenigstens seit der Zeit Friedrichs II. eine wahrscheinlich ständige, mit der Landvogtei durchans identische und auch das Elsaß betreffende Einrichtung. ${ }^{1}$ ) Aber auch abgesehen von der mangelnden Verknüpfung mit allgemeinen Erscheinungen ist es im Interesse einer Forschung, die nach präzisen Formulierungen strebt, zu bedauern, daB B. das von $\mathrm{jhm}$ selbst in den Regesten des Anhanges für die Verwaltung des

1) Bei anderer Gelegenheit gedenke ich auf diese Dinge nochmals ausführlich zurückzukommen. 